\title{
Erratum to: Structure and dynamics of the influenza A M2 Channel: a comparison of three structures
}

\author{
Hadas Leonov • Isaiah T. Arkin
}

Published online: 1 September 2010

(C) Springer-Verlag 2010

Erratum to: J Mol Model (2009) 15:1317-1328

DOI 10.1007/s00894-009-0493-2

The original version of this article unfortunately contained a mistake. The RMSD comparative values given in the 6th paragraph of the Introduction section were wrong. The correct text should be: (i) X-ray versus ssNMR: $4.0 \AA$; (ii) X-ray versus solution NMR: $4.0 \AA$ and (iii) ssNMR versus solution NMR: $4.6 \AA$. This error does not change any of the conclusions of the work.

The online version of the original article can be found at http://dx.doi. org/10.1007/s00894-009-0493-2.

H. Leonov $\cdot$ I. T. Arkin $(\bowtie)$

Department of Biological Chemistry,

The Alexander Silberman Institute of Life Sciences,

The Hebrew University of Jerusalem,

Edmund J. Safra Campus Givat-Ram,

Jerusalem 91904, Israel

e-mail: arkin@huji.ac.il 